\title{
Anabases
}

ANABASES Traditions et réceptions de l'Antiquité

$21 \mid 2015$

Varia

\section{Entre ici et ailleurs. Les enjeux des comparatismes}

Entretien avec Christian Jacob

\section{(2) OpenEdition}

\section{Journals}

Édition électronique

URL : http://journals.openedition.org/anabases/5326

DOI : $10.4000 /$ anabases.5326

ISSN : 2256-9421

\section{Éditeur}

E.R.A.S.M.E.

Édition imprimée

Date de publication : 1 avril 2015

Pagination : 197-211

ISSN : 1774-4296

\section{Référence électronique}

"Entre ici et ailleurs. Les enjeux des comparatismes », Anabases [En ligne], 21 | 2015, mis en ligne le 01 avril 2018, consulté le 21 octobre 2019. URL : http://journals.openedition.org/anabases/5326 ; DOI : 10.4000/anabases.5326 


\title{
Entre ici et ailleurs. \\ Les enjeux des comparatismes
}

Entretien avec Christian JACOB ${ }^{1}$

\begin{abstract}
hristian Jacob est directeur de recherche au cNRS et directeur d'études à l'École des Hautes Études en Sciences Sociales (Paris). À partir de travaux consacrés à l'histoire et à l'épistémologie de la géographie antique (L'Empire des cartes, I992; Géographie et ethnographie en Grèce ancienne, Iggr), il a développé des recherches sur la constitution des savoirs, dans une perspective comparatiste, dans le temps et dans l'espace. Le cheminement et l'aboutissement, encore à venir, de ce programme ouvert et ambitieux sont notamment constitués par l'entreprise collective des Lieux de savoir, sous la forme de deux imposants volumes: I. Espaces et communautés, 2007; II. Les mains de l'intellect, 20II. Il privilégie toujours davantage une approche "qui consiste à travailler sur des situations, des contextes d'échanges et de contacts interculturels». L'analyse des différentes formes de diffusion et de réception de l'Antiquité constitue un terrain particulièrement fécond.
\end{abstract}

Pascal Payen: Il n'est pas aisé de présenter les cheminements qui ont donné lieu à l'œuvre de Christian Jacob. Il s'agit d'une œuvre à la fois abondante et complexe, pour laquelle vous n'avez jamais hésité à vous engager dans des renouvellements venus d'autrui, ou bien dans des hypothèses nouvelles, issues des cheminements de votre propre réflexion, de vos propres avancées. Pour présenter l'ensemble de votre œuvre, je retiendrai deux moments forts dans vos travaux, à travers quatre livres.

1 Ce texte, fruit d'un entretien dans le cadre du séminaire de l'équipe erasme (université Toulouse-Jean Jaurès), a été retranscrit et annoté par Anthony Andurand, révisé par Corinne Bonnet et Pascal Payen, revu par Christian Jacob. 
Il y eut tout d'abord, pourrait-on dire, un premier moment où s'est forgé l'historien de la géographie ancienne, à partir des apports, notamment, de la philologie et de l'anthropologie. Cette période correspond, à mon sens, à trois grandes productions. D'abord, une monumentale thèse d'État, Géographie et culture en Grèce ancienne ${ }^{2}$, soutenue en ig87. Il s'agit d'une vaste histoire de la géographie et de la cartographie des Grecs, à travers l'analyse de l'œuvre singulière de Denys d'Alexandrie, en II87 vers, qui a servi de manuel de géographie jusqu'au début du XVIII ${ }^{\mathrm{e}}$ siècle pour le monde cultivé. Denys est un compilateur, qui a tout lu, depuis Homère et Hérodote jusqu'aux géographes alexandrins, et qui tient compte de l'extension du monde connu à l'époque même où il vit, celle d'Hadrien. Cette époque est celle de la mise en place d'une vision universaliste, qui embrasse d'un seul tenant Grecs et Barbares. Autrement dit, le clivage sur lequel s'était formée une part de la réflexion des Grecs de l'époque classique, la séparation si radicale entre Grecs et Barbares, n'a plus le même sens et tend en large part à s'effacer. Pour comprendre la signification de ce texte, du genre dans lequel il s'inscrit, il fallait prendre en compte toute l'histoire culturelle qui se rapporte à la description de la terre habitée et à la géographie - c'est notamment l'objet de la troisième partie de votre travail. Mais ce qui est singulier dans ce travail, et qui l'était encore davantage au moment où vous l'avez conduit, est que l'ensemble de la thèse se présente comme une réflexion sur la théorie de la description de la terre habitée et de la carte qui sous-tend le travail de Denys. En d'autres termes, vous ne cessez de vous interroger sur les catégories mentales des Grecs et sur leur capacité à extraire des modèles, à partir de la réalité, à construire des abstractions à partir de la diversité du réel qui les entoure.

Cette expérience forte a été ensuite élargie, approfondie, dans sa dimension spatiale et comparatiste, dans un livre qui fait toujours référence, $L^{\prime} E m p i r e$ des cartes $^{3}$. Dans ce livre, il n'est plus question seulement des Grecs: on les quitte, dans une vision large sur le plan spatial et théorique, mais pour essayer ensuite de revenir vers eux, pour mieux les mettre en perspective au terme d'un long détour. J'en lis un passage, qui résume bien, à mon sens, l'ensemble de votre méthode et de ces deux premiers livres:

«Notre fil conducteur sera une phénoménologie de la perception cartographique: reconstituer les itinéraires du regard, les points prégnants de la carte susceptibles de le fixer, les voies d'accès, directes ou tortueuses, aux informations géographiques principales ou secondaires, la hiérarchisation et l'étagement des niveaux de vision,

2 C. JАсов, Géographie et culture en Grèce ancienne: essai de lecture de la Description de la terre habitée de Denys d'Alexandrie, Lille, ANRT, I989.

3 C. ЈАсов, L'Empire des cartes: approche théorique de la cartographie à travers l'histoire, Paris, Albin Michel, I992. 
déterminés par les choix graphiques de l'auteur, les accentuations chromatiques, la répartition des ornements, des inscriptions et des éléments figuratifs. Car la carte ne se réduit pas au dessin topographique; ses autres composantes, loin de jouer un rôle accessoire, conditionnent le regard et l'orientent selon des modalités qu'il nous faut définir ${ }^{4}$.»

Le troisième livre issu de cette première étape de vos recherches est un admirable petit classique, aujourd'hui malheureusement introuvable: Géographie et

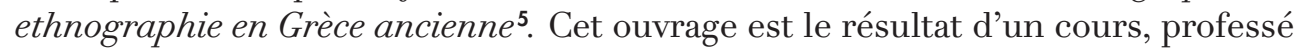
devant les étudiants de Vincennes, mais aussi la synthèse des deux premiers livres que nous avons évoqués. C'est une histoire de la géographie, mais aussi de l'ethnographie dans le monde grec, dans laquelle vous vous demandez comment les hommes habitent un territoire, comment ils l'occupent avec d'autres et comment, à partir du rapport au territoire, un peuple peut construire sa propre identité, voire ses identités multiples, au contact des autres.

Ainsi, pendant une bonne dizaine d'années, à partir de ces trois livres, on voit se dessiner une forme de pensée originale, et cette originalité, selon moi, vient de ce que les questions que vous posez portent à la fois sur les détails matériels les plus concrets qui soient, mais aussi sur la manière propre, pour les Grecs ou pour les Barbares, de concevoir le monde. Ces observations m'inspirent deux questions: est-ce cela que vous appelez «anthropologie historique», à votre manière? Quels sont, par ailleurs, les auteurs, quelles sont les références théoriques sur lesquels vous vous êtes appuyé, dans les années ig8o et jusqu'au début des années i99o, pour mener à bien ces travaux?

Christian Jacob: Merci beaucoup pour ces réflexions. C'est un moment extrêmement précieux et intimidant, qui - au-delà de l'aspect «notice nécrologique »--, permet en tout cas de réfléchir à la cohérence d'un travail, d'un cheminement. Je réponds donc volontiers à vos deux questions.

Oui, le premier moment que vous avez évoqué peut être décrit comme celui d'une «anthropologie historique». Encore en amont de la thèse et de ce travail, il y a eu une étape, restée pratiquement inédite - hormis peut-être un article sur la Périégèse de Pausanias ${ }^{6}$-, un travail expérimental sous la direction de Marcel Detienne, influencé, aussi, par un professeur d'archéologie de Paris Iv, Philippe

\footnotetext{
$4 \quad$ Ibid., p. I43.

5 C. ЈАсов, Géographie et ethnographie en Grèce ancienne, Paris, Armand Colin, I99I.

6 C. ЈАсов, “Paysages hantés et jardins merveilleux: la Grèce imaginaire de Pausanias », L'Ethnographie, Lxxvi, n.s. I (I980), p. 35-67; “The Greek traveler's areas of knowledge: Myths and other discourses in Pausanias' Description of Greece », Yale French Studies 59 (I980), p.65-85.
} 
Bruneau, qui a été le codirecteur de ma maîtrise. Dans ce premier travail, j'hésitais encore entre deux modèles, celui d'un formalisme sémiotique inspiré de Greimas qui représentait alors, pour les jeunes chercheurs désireux d'intégrer les dernières nouveautés de la place parisienne, le nec plus ultra en termes de «machine intellectuelle -, et celui que proposaient Vernant et Detienne, dont, dès cette époquelà, j'ai saisi l'intérêt, la portée et les possibles implications, pour l'étude non plus simplement de la cité, de la religion, des pratiques symboliques et du sacrifice, mais aussi des modes de fonctionnement des savoirs et de l'univers mental des Grecs. Une autre influence, paradoxale sans doute mais qui revient périodiquement, est celle d'Ignace Meyerson. Jean-Pierre Vernant m'a souvent confié que, parmi ses élèves de la deuxième ou troisième génération, j'étais un des seuls qui avait lu Meyerson et le relisait périodiquement: une façon de dire mon intérêt pour les facettes d'un rapport au monde, à l'espace, au temps, à la mémoire, à la raison, déterminé historiquement et culturellement, et pouvant donc se traduire dans des communautés, des pratiques ou des généalogies savantes.

Cette anthropologie historique m'a conduit à m'intéresser à ce qui était secondaire chez Vernant et Detienne, c'est-à-dire cette tradition savante qui m'a fasciné dès le départ, ces scholiastes, ces lexicographes, la Souda, les paradoxographes, ces auteurs souvent mineurs, mais dont Vernant et Detienne montraient qu'ils étaient les vecteurs à la fois d'une encyclopédie, d'une bibliothèque, de cadres de pensée, d'une mémoire, transformés sous la forme de notices lexicographiques, de bribes de commentaires, de notices géographiques, etc. Je me suis donc orienté vers ce qui, du monde grec, n'était pas pris en charge par Vernant, Detienne ou même Vidal-Naquet, à savoir les bibliothèques savantes et les géographes, qui mobilisaient à la fois des représentations du monde, mais aussi des pratiques d'écriture ou de visualisation, la constitution de généalogies savantes, la confrontation de visions du monde concurrentes, comme celle des géomètres alexandrins et celle des géographes littéraires, héritiers d'Homère et d'Hésiode.

Au chapitre des références théoriques, donc: Meyerson, dont je n'ai pas repris toute l'armature théorique, est quelqu'un que j'ai croisé dans les dernières années de sa vie, sans pleinement réaliser l'urgence qu'il y aurait eu à assister à tous ses séminaires. Une autre référence déterminante a été celle de Michel de Certeau, rencontré alors j'étais encore doctorant, qui m'introduisit dans ce milieu des colloques de sémiotique à Urbino, où l'on croisait des penseurs comme Umberto Eco, qui n'était pas encore la star qu'il est devenu depuis, Jean-François Lyotard, Hubert Damisch, Louis Marin, c'est-à-dire un creuset de chercheurs anti-mandarins, qui accordaient autant d'importance au dialogue avec un étudiant thésard qu'avec des gens de leur milieu. Une sorte de milieu intellectuel dans lequel de Certeau se dégageait, pour plusieurs raisons, à savoir l'inspiration insufflée à mes recherches sur l'histoire de la cartographie, l'histoire des voyages et l'historiographie, mais aussi une leçon qui m’a profondément marqué et que j’essaie 
de transmettre à mon tour à mes étudiants : les chercheurs sont des braconniers, et les meilleurs braconniers sont ceux qui n'hésitent pas à sauter par-dessus les clôtures pour suivre le lièvre, d'un champ à l'autre. La leçon de Michel de Certeau à ses jeunes interlocuteurs était la suivante: «Soyez mobiles, ne restez pas dans un terrain unique; vos objets de recherche peuvent vous conduire à franchir les frontières de certaines disciplines. » Cette forme de liberté intellectuelle-chose que je précise aussi à mes étudiants - a été rendue possible par mon recrutement au cNRs, où l'on peut, ce n'est pas négligeable, justifier de passer des études grecques à l'histoire de la géographie, de l'anthropologie des savoirs aux sciences cognitives. C'est peut-être plus difficile dans le cadre d'un ancrage dans un département d'histoire. Une autre référence théorique, concernant l'histoire de la cartographie: les américains, comme J.Brian Harley et David Woodward, qui ont été les maîtres d'œuvre d'une monumentale histoire de la cartographie ${ }^{7}$ publiée par les Presses de l'université de Chicago, avec un appel d'air extraordinaire, la mobilisation d'un vaste panel d'auteurs internationaux, une grande innovation intellectuelle. Malheureusement, le premier volume était déjà très largement entamé lorsque j’ai fait la connaissance de ces professeurs. Ils ont en fait traduit en anglais un certain nombre de textes - dus à Germaine Aujac ${ }^{8}$, par exemple -, et, d'une certaine façon, c'est un peu l'école française qui se trouvait impliquée dans ce projet. Voilà donc pour cette première phase, en termes d'influences intellectuelles.

Pascal Payen: Jevoudrais maintenantvous interroger sur un deuxième domaine, que j’ai annoncé précédemment, celui qui est illustré par la vaste entreprise des Lieux de savoir 9 . On peut définir cette entreprise comme une histoire comparée des pratiques intellectuelles, qui représente une sorte d'aboutissement, provisoire du moins, dans le cheminement intellectuel que nous venons d'évoquer. C'est une vaste entreprise que vous avez endossée, conçue, écrite, en partie, que vous avez fait se développer et que vous continuez à diriger. Des quatre volumes initialement prévus, les deux premiers ont déjà paru au format papier, les deux autres paraîtront sous une autre forme. Il est hors de question de résumer ici, même brièvement, ces deux volumes. Je voudrais simplement vous poser un faisceau de questions, qui

7 J. B. Haley, D. Woodward (ed.), The Historyof Cartography, London-Chicago, University of Chicago Press, ig87-... (4 vol. à ce jour).

8 G. Aujac, "The Foundations of Theoretical Cartography in Archaic and Classical Greece ", "The Growth of an Empirical Cartography in Hellenistic Greece ", "Greek Cartography in the Early Roman World », in J. B. Haley, D. Woodward (ed.), The History of Cartography, London, Chicago, University of Chicago Press, I987, I, p. I3o-176.

9 C. JАсов (dir.), Lieux de savoir. I: Espaces et communautés, Paris, Albin Michel, 2007; Lieux de savoir. Les mains de l'intellect, Paris, Albin Michel, 2oIr. Carnet de recherche accessible sur la plateforme hypotheses.org: lieuxdesavoir.hypotheses.org/ 
font sens et signe pour les chercheurs que nous sommes, jeunes ou moins jeunes. D'abord, quel bénéfice retirez-vous d'une telle expérience, dans sa dimension collective? Sont-ce au contraire, au terme du parcours, les obstacles et les limites que vous retenez et qui l'emportent? Ensuite, pour élargir quelque peu le propos, la recherche, telle que nous la pratiquons maintenant, est-elle “condamnée» aux vastes projets collectifs? De quel côté, pour terminer, incline votre penchant de chercheur, quel est selon vous le milieu le plus propice aux découvertes? Est-ce lorsqu'on est seul devant la page blanche, crayon en main, ou bien la position de celui qui, ouvrant son ordinateur, se trouve connecté avec des milliers de savants? Vers quelle position va votre préférence?

Christian Jacob: Quelques mots, tout d'abord, sur les bénéfices d'une expérience collective comme celle des Lieux de savoir. Le modèle a été les grandes entreprises comparatistes menées par Vernant et Detienne, les volumes sur les problèmes de la guerre, de la terre, de la divination en Grèce ancienne, sur les savoirs de l'écriture ${ }^{10}$, ou le volume de Detienne sur les tracés de fondation ${ }^{11}$. Je crois que c'est Vernant qui a le mieux parlé de ces formes de communauté savantes, électives, créées, le plus souvent, en dehors des institutions, sur la base d'une camaraderie intellectuelle, de liens d'amitié et de complémentarité, et au sein desquelles chacun accepte d'apporter la technicité de son travail et de son expertise, de ses dossiers de sources premières, mais aussi d'entrer dans un jeu de communication avec des spécialistes de tout autres domaines. Je dirais donc que, pour moi, ce type de travail collectif est une des raisons d'être de la recherche en sciences humaines, à savoir partager des questions, construire des objets, être, aussi, dans une démarche évolutive: les Lieux de savoir ont ainsi connu des étapes antérieures, sous forme de colloques, de journées d'étude, de séminaires, d'ateliers, soit tout un ensemble d'étapes qui permettent de construire peu à peu un réseau d'amitié, de connivence, d'utopie et de déraison, aussi, puisque on pourrait imaginer que ce type d'entreprise scientifique, avec son lot de difficultés techniques, économiques, logistiques, puisse ne pas être retenu par des chercheurs d'un tempérament différent. L'avantage, selon moi, c'est que ce type d'entreprise collective permet de soulever des montagnes, si je puis dire. On peut très bien

10 J.-P. Vernant (dir.), Problèmes de la guerre en Grèce ancienne, Paris-La Haye, Mouton, Ig68; M. I. Finley (dir.), Problèmes de la terre en Grèce ancienne, Paris-La Haye, Mouton, i973; J.-P. Vernant et al., Divination et rationalité, Paris, Le Seuil, i974; M.Detienne, J.-P.Vernant, Les ruses de l'intelligence: la mètis des Grecs, Paris, Flammarion, i974; M. Detienne, J.-P. Vernant, La cuisine du sacrifice en pays grec, Paris, Gallimard, I979; M. Detienne (dir.), Les savoirs de l'écriture en Grèce ancienne, Lille, Presses universitaires de Lille, 1988.

1 M. Detienne (dir.), Tracés de fondation, Louvain-Paris, Peeters, I99o. 
choisir de travailler seul et d'être un architecte, un bâtisseur tout à fait performant sur un terrain déterminé; mais les Lieux de savoir, en mobilisant une quarantaine d'auteurs pour chaque volume, en les obligeant à se lire les uns les autres et à réfléchir les uns par rapport aux autres, ont permis de déblayer un énorme terrain, et surtout de faire se rencontrer des mondes, plus ou moins éloignés, qui ne communiquent pas toujours : le monde des antiquisants, le monde des anthropologues, le monde des historiens modernes, des historiens des sciences, des sinologues ou des spécialistes du monde arabe. Il s'agissait, aussi, d'entrecroiser leurs expertises sur un jeu de questionnements comparatistes : qu'est-ce que les savoirs? De quel type sont les pratiques qui produisent du savoir? Comment certains énoncés, certains artefacts sont identifiés comme «savants » dans nos sociétés? Quels sont les acteurs individuels, les communautés, quels sont les lieux ou les dynamiques spatiales qui permettent la production et la diffusion de ces artefacts? Un tel ensemble de questions gagne, je crois, à être éclairé, travaillé d'une manière très expérimentale, c'est-à-dire sans souci d'exhaustivité ou d'encyclopédisme, en adoptant souvent le parti pris de la distance maximale, comme lorsque l'on confronte l'exploration du sol de la planète Mars par les rovers téléguidés de la NASA ${ }^{12}$ et les pratiques de mobilité de la cour iranienne ${ }^{13}$ : ce sont des univers hautement distincts, et lorsqu'on rajoute comme troisième terme les récits d'exploration ethnographique en Afrique au début du $x^{e}$ siècle $^{14}$, on obtient une triangulation qui oblige à penser le rapport aux lieux, le rapport à la distance, à la sphère d'action, le statut du passage, du voyage, du déplacement ou de l'ancrage, de l'ici et ailleurs, et on parvient à faire avancer la réflexion collective sur des sujets de ce type-là.

Sur le deuxième aspect de votre question, je dirai qu'évidemment un projet comme les Lieux de savoir s'est appuyé sur une infrastructure “légère», celle d'un Groupement de Recherche International (GDRI) du CNRS, au sein duquel nous avons pu, en quelques années, organiser quantité de colloques, de tables rondes, de séminaires, avec des invités venus de partout, dont j'ai dû assurer moi-même la coordination - le financement de pareils projets ne prévoit que rarement les moyens humains nécessaires à leur réalisation. À un certain moment, cette infrastructure s'est arrêtée; nous n'avons pas demandé de renouvellement car les inconvénients logistiques, le poids gestionnaire devenaient un peu paralysants par rapport à l'avancée du projet intellectuel. Nous avons donc adopté des manières

12 E. Benazera, “Structures et pratiques du savoir à distance: le cas de l'exploration robotique de Mars », in Lieux de savoir, I, p. 897-915.

13 Z. Vesel, F. Richard, “La culture de cour dans le monde iranien de l'époque sassanide aux Qadjars », in Lieux de savoir, I, p. 854-874.

14 É. Jolly, «La mission ethnographique Dakar-Djibouti: collecte itinérante et maîtrise du terrain ", in Lieux de savoir, I, p. 875-895. 
de travailler tout aussi efficaces, mais décentralisées, reposant davantage sur les échanges par internet - d'où, aussi, la mutation du projet aujourd'hui. Plus récemment, j'ai contribué à la création d'un Laboratoire d'Excellence (LabEx) à Paris, “Histoire et anthropologie des savoirs, des techniques et des croyances ${ }^{15}$ » (HASTEC). J'ai consacré beaucoup de temps et d'enthousiasme à cette initiative, dont je reste proche, mais je me rends compte maintenant qu'il y a des logiques gestionnaires, bureaucratiques, administratives très importantes, qui demandent que les chercheurs acceptent de sacrifier beaucoup de leur temps. Ce type de projet en vaut la peine, car cela permet de financer des contrats doctoraux, des post-doctorants et de sortir de la difficulté des jeunes chercheurs, mais je pense qu'il doit vraiment y avoir une rotation à ce genre de fonction, de responsabilité. D'abord, dans un souci de démocratie, d'ouverture, de pluralisme intellectuel, mais aussi parce qu'il convient de préserver le temps du travail personnel.

Pour répondre, enfin, à votre dernière question, sur la solitude du travail intellectuel et le travail connecté, Tzvetan Todorov prétendait il y a quelques années que, finalement, la nouvelle administration du travail de la recherche (en projets, appels d'offre, laboratoires, etc.) était une hérésie, parce que le chercheur en sciences humaines a seulement besoin d'un ordinateur chez lui et de l'accès à une bibliothèque. S'agissant de ma propre pratique, cependant, je ne conçois pas le travail de recherche comme complètement isolé ou déconnecté; les séminaires jouent un rôle très important, comme lieux de mise à l'épreuve des premières hypothèses, des premiers résultats, comme lieux, aussi, de questionnement. Les meilleurs séminaires sont ceux où l'on n'hésite pas à aller au-delà de sa pensée, à prendre des risques intellectuels en disant des choses que l'on n'écrirait pas nécessairement immédiatement; c'est une manière de tester, de soumettre à la critique et à l'intelligence collective. On ne peut donc travailler seul, mais on peut travailler en réseau. J'ai découvert Twitter assez récemment, il y a deux ans. C'est devenu une extension de mon bureau: il ne s'agit pas seulement de faire état de son quotidien, mais aussi de demander à ses collègues, tard dans la soirée, la copie numérique de la Bibliotheca Universalis de Gesner et de recevoir quelques heures plus tard les informations nécessaires pour y accéder en ligne. C'est donc un lieu d'expertise extraordinaire, une manière de séminaire décentralisé, un lieu d'invention, d'ébullition intellectuelle, conceptuelle, bibliographique, qui peut faire perdre, mais aussi gagner beaucoup de temps.

Corinne Bonnet: Nous venons d'évoquer les noms de Detienne et de Vernant, qui seront l'occasion pour moi de revenir sur la pratique du comparatisme, qui fait une part de l'intérêt et de l'originalité de votre œuvre. Le comparatisme séduit 
aussi nos étudiants, qui viennent vers nous avec des sujets de mémoire qui vont de l'Égypte à la Mésopotamie, voire avec des choses plus exotiques encore. Le comparatisme attire, et c'est une démarche, je dirais, assez «naturelle». Dans une de vos publications, vous écrivez que le comparatisme est un peu votre «tribu ${ }^{16}$ », à laquelle je me rallierais aussi moi-même volontiers. Dans le même temps, le comparatisme est un exercice difficile, risqué. Puis-je vous demander quel bilan vous faites de ces expériences comparatistes? Est-il vraiment possible de comparer des cultures aussi différentes que celles de la Chine et de la Grèce, du Japon et de Rome, en faisant le va-et-vient, aussi, entre des époques très différentes? Quelles sont les précautions, quels sont les avantages ou les inconvénients?

Christian Jacob: Effectivement, il existe plusieurs manières de pratiquer le comparatisme. On peut le pratiquer seul : c'est ce que fait quelqu'un comme Geoffrey Lloyd ${ }^{17}$, qui a appris suffisamment de chinois classique pour accéder directement à certains textes et offre ainsi une mise à plat de certains questionnements, concernant le monde grec, le monde mésopotamien ou le monde chinois. Il y a aussi un comparatisme comme celui de François Jullien ${ }^{18}$, à la fois sinologue et helléniste, qui, contrairement à Lloyd, ne cherche pas la production d'un savoir historique ou anthropologique "objectif», mais voit dans le comparatisme une manière de faire de la philosophie: approche très suggestive intellectuellement, mais dans le même temps très difficile à appliquer en tant que méthode. C'est une approche si personnelle, si individualisée, qu'on a du mal à voir comment s'approprier un tel outil. Le comparatisme de Vernant et de Detienne est un comparatisme collectif, qui passe par le dialogue avec des spécialistes et nécessite, pour les membres de cette “tribu», le désir d'en faire un peu plus: non seulement écouter, lors de séminaires, ce qu'aura à dire le collègue spécialiste d'un autre domaine, mais aussi entamer une réflexion plus élaborée entre ici et ailleurs et, sans prétendre à la compétence d'un spécialiste d'une autre ère culturelle, essayer

16 C. ЈАсов, “Anthropologie, comparatisme: expérimenter sur les savoirs »(Conférence SFHSH 3, 4 février 20II), en ligne: http://lieuxdesavoir.hypotheses.org/8I3

17 G. E. R. Lloyd, Ancient Worlds, Modern Reflections: Philosophical Perspectives on Greek and Chinese Science and Culture, New York-Oxford, Oxford University Press, 2004; The Delusions of Invulnerability: Wisdom and Morality in Ancient Greece, China and Today. London, Duckworth, 2005; Principles And Practices in Ancient Greek And Chinese Science, Aldershot, Ashgate, 2006.

18 F. Jullien, La Valeur allusive. Des catégories originales de l'interprétation poétique dans la tradition chinoise, Paris, École française d'Extrême-Orient, I985; La Chaîne et la trame. Du canonique, de l'imaginaire et de l'ordre du texte en Chine, Paris, PUF, 2004 ( ${ }^{\mathrm{re}}$ éd.: Paris, Presses universitaires de Vincennes, I986); De l'universel, de l'uniforme, du commun et du dialogue entre les cultures, Paris, Fayard, 2008. 
de s'approprier certaines des questions, certains des objets que ce spécialiste a construits, pour les appliquer à ses propres objets. Dans mon cas, c'est vrai que le monde chinois est un univers où je voyage périodiquement, au sens propre comme au sens figuré. Nous y sommes allés avec Marcel Detienne il y a deux ans - l'un de ses élèves a en effet créé le département d'histoire ancienne à l'Université Fudan de Shanghai -, et j'y suis retourné en octobre pour enseigner Hérodote, Plutarque, Athénée à des étudiants tout à fait enthousiastes et passionnés. Pourquoi la Chine? D'abord, pour des raisons et des rencontres personnelles: Jean-Pierre Drège, spécialiste de l'histoire du livre et des bibliothèques, et Anne Cheng, maintenant professeur au Collège de France, spécialiste de l'histoire de la pensée chinoise. La collaboration avec Anne Cheng va assez loin, puisque j'ai déjà été dans le jury de thèse de trois de ses étudiants ; je suis également invité, par son intermédiaire, à des écoles d'été sinologiques et viens de terminer un texte, à paraître dans les actes d'un colloque ${ }^{19}$ sur un classique chinois, le Da xue ou “Grande Étude», dans lequel j’apporte un point de vue complètement extérieur sur ce texte. Ce type de comparatisme demande d'abord un effort conséquent d'immersion: il faut lire, aller au-delà de la musique de noms propres ou d'œuvres complètement exotiques; il faut essayer de saisir et de s'approprier les enjeux d'une histoire. Le travail sur le Da xue porte sur un texte extrêmement court, qui était intégré dans le corpus des classiques de Confucius à très haute époque et qui est devenu, en quelque sorte, une introduction au confucianisme encore valable aujourd'hui. Pour le présenter très brièvement, c'est un texte qui fait l'objet d'une tradition exégétique extraordinaire, multiséculaire, au cours de laquelle on a travaillé à la fois sur sa littéralité et sur sa structure - le texte était à l'origine écrit sur des lamelles de bambou, un support fragile, qui par la dissociation éventuelle des lamelles, peut également perturber l'agencement d'un texte. C'est également un texte à l'intérieur duquel des commentaires sont venus se glisser progressivement. Il permet donc de penser l'ensemble des opérations sur lesquelles peut s'appuyer la fabrique d'un texte en tant qu'objet intellectuel, sur la façon dont il devient un champ de problèmes, sur la façon de déterminer une marge d'action et un espace d'intervention qui permettent à certains lecteurs autorisés d'entreprendre une série d'opérations techniques, intellectuelles, herméneutiques. C'est donc un lieu privilégié de comparaison avec les pratiques des philologues alexandrins sur le texte homérique, en pointant immédiatement les différences entre les deux corpus et en m'intéressant également à des modes de positionnement tout à fait différents. Ainsi les philologues alexandrins procèdent à des formes d'édition non destructives des textes, sous la forme de signes marginaux, tandis que leurs

19 “Lectures et usages de la Grande Étude», colloque international organisé par la chaire d'Histoire intellectuelle de la Chine, Paris, Collège de France, 23-24 juin 20 Ir. 
collègues chinois n'hésitent pas à copier, coller, reconfigurer, réécrire et à rechercher des nouvelles formes de textualité qui permettront de dire, mieux que le texte original, ce qu'il a voulu signifier, au point d'aboutir parfois à des diagrammes et à des schémas complètement abstraits. Par cet exemple précis, on voit qu'il ne s'agit pas de dresser, par le comparatisme, un immense panorama, ni d'établir l'inventaire, terme à terme, des différences et des similitudes, mais plutôt de comprendre des scénarios culturels très particuliers, comme cette tradition exégétique liée à un classique chinois sur plusieurs siècles, d'essayer de repérer les logiques, les positionnements, les artefacts qui sont produits, les modes de raisonnement, les controverses, et de comparer cela avec ce qui est produit dans le Musée d'Alexandrie ou à Pergame sur le texte homérique, à savoir des paradigmes de lectures qui entrent en concurrence, des controverses très vives - le texte comme surface ou au contraire comme profondeur, pour ne citer qu'un exemple. Voilà donc un type possible d'enquête comparatiste selon moi.

Un autre axe de mon travail actuel concerne, dans le cadre d'une réflexion sur la construction des traditions, les travaux des cognitivistes, sur la manière dont la culture peut être transmise d'individu à individu, de génération à génération. Ce sont des manières de penser qui me sont très étrangères, mais qui posent des questions très intéressantes, y compris pour un spécialiste du monde grec. On peut être amené à comparer la transmission des ritournelles dans les cours de récréation des écoles primaires avec la transmission culturelle des poèmes homériques dans le monde grec... Le comparatisme est donc une manière de penser les terrains sur lesquels on travaille, en utilisant des éclairages qui peuvent certes présenter des similitudes, mais se situer aussi dans l'éloignement maximal. Je crois que c'est Marcel Detienne qui m’a inspiré à ce propos une image, que je réutilise parfois auprès de mes étudiants: il peut être intéressant de mettre dans le monde grec, comme on le fait dans un tube à essai au moyen d'une pipette, quelques " gouttes » de Chine, et d'analyser la réaction chimique ainsi produite. Le comparatisme, à ce titre, est une pratique très empirique.

Pascal Payen: Sur ce point, on voit aussi que le comparatisme est toujours une pratique exigeante, en ce qu'elle appelle des lectures, un effort de documentation sur un domaine qu'on ne connaît pas ou peu. Et la chose devient plus difficile encore quand la comparaison fait intervenir plus de deux termes.

Adeline Grand-Clément: On voit également, au-delà des lectures, l'importance des discussions - d'où l'intérêt des séminaires -, et donc aussi des relations d'affinité, et je dirais même de confiance avec les spécialistes du domaine concerné.

Christian Jacob: Une autre voie d'accès, peut-être plus aisée à mettre en œuvre, consiste à travailler sur des situations, des contextes d'échanges et de contacts 
interculturels. Plutarque, entre Grecs et Romains, en est un bon exemple. Dans le volume collectif sur ce classique chinois auquel j'ai fait allusion, de même, il y a un chapitre extraordinaire écrit par un père jésuite, Thierry Meynard (qui se trouve d'ailleurs en Chine actuellement), sur les premières traductions occidentales de ce texte, par les jésuites du Xvir ${ }^{\mathrm{e}}$ siècle, je crois. Ce petit traité a été le premier traité confucéen traduit en latin, et l'étude de cette traduction montre très concrètement comment les jésuites comparaient deux univers culturels très différents, l'univers confucianiste et l'univers aristotélico-thomiste occidental, en essayant de négocier chaque correspondance, chaque traduction, d'annoter, de créer des ponts et des passerelles, sur les significations philosophiques, théologiques, aussi bien que politiques. On voit là le comparatisme en œuvre: la traduction en latin, le lien entre les termes latins et les caractères chinois, ainsi que les commentaires ajoutés par les jésuites témoignent véritablement d'un laboratoire comparatiste tout à fait extraordinaire.

Corinne Bonnet: Une dernière question. Je voudrais lire un texte de votre plume, qui m'a beaucoup plu, pour aborder une question que nous avons l'habitude de poser à nos invités, et ce d'autant plus qu'Anabases est une revue qui se soucie de la réception de l'Antiquité et donc de la manière dont le présent “vit» l'Antiquité, s'en empare, l'interroge ou la rejette. Voici le passage en question:

«Je voudrais dire ma conviction forte dans l'importance et l'actualité de la recherche dans le champ des humanités et des sciences sociales. D'abord, chercher à comprendre l'univers d'un scribe mésopotamien, d'un encyclopédiste grec, d'un médecin de la Renaissance, d'un guérisseur africain ou d'un chaman amérindien présente un intérêt en soi, et on n'a pas à s'excuser ou à entrer dans la logique d'une justification utilitariste. Mais de plus, ce désir de comprendre l'humain dans la diversité de ses activités, de ses cultures, de ses sociétés et de ses productions symboliques, nous apporte un surplus d'intelligibilité, une distance critique, des leviers cognitifs et comparatistes pour nous comprendre nous-même, dans notre rapport au monde, aux techniques et aux savoirs. Il n'y a pas de sens sans un horizon ${ }^{20}$. »

Devant cette belle prise de proposition, à laquelle je souscris avec enthousiasme, quel est donc le présent de l'Antiquité, aujourd'hui, pour un antiquisant comme vous?

Christian Jacob: Je pense que les études anciennes, l'histoire, l'anthropologie, dans toute la diversité de leurs formes, les sciences sociales et les humanités d'une manière générale sont absolument indispensables aujourd'hui. Ce texte est aussi

20 C. ЈАсов, “Penser avec l'absent» (Conférence sfHsh 5, 4 février 20II), en ligne: http:// lieuxdesavoir.hypotheses.org/822 
une prise de position au sujet des propos d'un ancien président français, qui avait une attitude totalement négative par rapport aux humanités... C'est également une réflexion que j'ai menée avec mes camarades du centre Anthropologie et Histoire des Mondes Anciens (ANHIMA), puisque que, comme votre équipe sans doute, nous avons été pris dans l'urgence d'un nouveau projet quinquennal, qui a été l'occasion de réfléchir à l'utilité des études anciennes aujourd'hui. Ma réflexion personnelle et celle de mes collègues se rejoignent sur ce point pour dire que nous sommes sans doute des artisans d'intelligibilité, de réflexivité sur tout ce qui se passe aujourd'hui. Travailler sur Athènes, sur Rome ou sur Alexandrie, sur la tradition européenne ou chinoise permet de poser des questions sur le politique, la société, le genre, sur le statut de l'étranger, la culture, la religion, le rapport au monde, le vivre ensemble, soit tout un ensemble de dimensions qui sont fondamentales dans les sociétés humaines. Cela veut dire deux choses: d'une part, que notre travail comporte une exigence de continuité et de collectivité-il faut continuer à écrire des livres, à organiser des colloques ou des séminaires, à publier des revues, à éditer et commenter des textes; d'autre part, il faut aussi porter notre travail et notre savoir vers la société civile, le monde politique, vers le monde de l'action sociale, aussi: éducateurs de rue, intervenants qui s'occupent des toxicomanes, des personnes à mobilité réduite ou atteintes de la maladie d'Alzheimer. Dans ce domaine, j'ai fait ces dernières années de très belles rencontres. Ces professionnels ont organisé il y a quelque temps un colloque européen sur le thème de la transmission, à l'occasion duquel ils m'ont invité à proposer le point de vue d'un historien et anthropologue sur cette notion. Ce fut une rencontre absolument inoubliable, très marquante pour moi, où j'ai pu voir le retour de cette société civile, engagée dans le concret très quotidien, sur les questions autour desquelles je travaille, à savoir le statut de la mémoire, de la culture, de l'oubli, de l'apprentissage, la nature du lien social, etc. Je plaide donc pour un rapport complètement décomplexé avec le savoir: travailler sur la Grèce ou Rome n'est pas plus stupide que de travailler en laboratoire sur la génétique de telle ou telle catégorie de mouches. Ce sont des formes de recherche scientifique très fondamentale, mais je crois que nous avons aussi, à notre époque, un devoir particulier pour éclairer le présent.

Mon dernier mot sur ce point sera lié à mon implication dans le domaine de l'histoire des savoirs et des pratiques savantes. Les travaux que je mène m'ont valu, à la demande du président de l'EHess, de participer comme observateur à une mission sur les humanités numériques - bien que je ne sois en aucune façon un spécialiste des nouvelles technologies. Et effectivement, le numérique, envisagé dans le long terme, modifie profondément l'enseignement, notre rapport à la recherche, notre rapport aux sources, modifie même nos univers de pensée: contrairement à ce que peuvent penser nos étudiants ou certains de nos collègues, qui considèrent parfois le numérique uniquement d'un point de vue instrumental et comme un outil destiné à faciliter nos tâches, je pense que nous assistons à des 
mutations extrêmement profondes de nos manières de penser, de lire, d'écrire, de visualiser, d'imaginer. Si l'on remonte un peu dans le temps, l'apparition du Thesaurus Linguae Graecae sous un format numérique avait déjà fait exploser les cadres de l'hellénisme traditionnel - plus de cent millions de mots grecs à portée de l'étudiant ou du chercheur! Aujourd'hui, à l'heure de la Text Encoding Initiative et des modes de visualisation de données, on peut poser à des textes ou à des bibliothèques des questions qui auraient été impensables auparavant - je pense à Athénée en particulier. Nos études sont donc tout à fait actuelles, et très importantes pour former de jeunes chercheurs et leur assurer un avenir.

Marion Maisonobe, doctorante en géographie: Quel a été, pour revenir à la question des influences, le rôle des géographes dans la construction de votre démarche et de votre travail?

Christian Jacob: Il y a eu une très belle rencontre avec un géographe français qui s'appelle Paul Claval. Il était à mon jury de thèse, et il a créé la revue Géographie et cultures ${ }^{21}$, qui existe toujours. Il faut aussi mentionner les Américains dont j'ai parlé, Brian Harley et David Woodward, qui étaient des géographes critiques, très marqués par le déconstructionnisme français (Foucault, Derrida...). J'ai été influencé par Roger Brunet et son travail sur la chorématique et les visualisations géographiques ${ }^{22}$. Actuellement, je suis intéressé par les travaux d'historiens de la géographie et de la cartographie, comme ceux de Jean-Marc Besse ${ }^{23}$ ou de Giorgio Mangani ${ }^{24}$. Et il y a bien sûr le courant que l'on dénomme le «tournant spatial », qui mobilise des concepts géographiques pour les appliquer à différents objets des humanités et des sciences sociales : la géographie peut offrir de très utiles paradigmes pour l'histoire et l'anthropologie des savoirs, et le projet même des «Lieux de savoir» en est le meilleur exemple...

21 La revue est accessible sur revues.org: http://gc.revues.org/

22 R. Brunet, “La composition des modèles dans l'analyse spatiale», L'Espace géographique 4 (I98o), p. 253-264; “La carte-modèle et les chorèmes », Mappemonde 4 (Ig86), p.2-6.

23 J.-M. Besse et al. (dir.), Du milieu à l'environnement: pratiques et représentations du rapport homme/nature depuis la Renaissance, Paris, Economica, I992; J.-M.Besse, Les grandeurs de la Terre: aspects du savoir géographique à la Renaissance, Lyon, ens Éd., 2003; J.-M. Besse et al. (dir.), Naissances de la géographie moderne, I760-I860: lieux, pratiques et formation des savoirs de l'espace, Lyon, ENs Éd., 2010.

24 G. Mangani, Cartografia morale: geografia, persuasione, identità, Modena, F. C. Panini, 2006; Geopolitica del paesaggio. Storie e geografie dell'identità marchigiana, Ancona, Il lavoro editoriale, 2012. 
Un étudiant: Comment la recherche universitaire, précisément, pourrait-elle davantage s'ouvrir en direction de la société civile et d'un public plus large?

Christian Jacob: L’une des idées que nous avons eues avec le LabEx HAstEc créé à Paris était de fonder un collège des savoirs, c'est-à-dire un lieu, qui pourrait être itinérant et proposer des échanges, des conférences, des écoles d'été, des partenariats avec des entreprises ou des associations. Malheureusement, les choses sont ainsi faites que les évaluateurs nous ont donné pour mettre en place ce dispositif une dotation très inférieure à celle qui était prévue, et ce collège a été supprimé, afin de privilégier des emplois pour des doctorants et des post-doctorants. Une autre voie serait de favoriser des passerelles entre la recherche, l'enseignement supérieur et l'enseignement secondaire. J'ai eu l'occasion de rencontrer des professeurs de lettres du secondaire, travaillant sur l'usage des nouvelles technologies dans les collèges et les lycées. J'ai été impressionné par leur dynamisme, leur originalité, leur inventivité, et en même temps par la demande très forte pour l'implication des chercheurs et des universitaires. La suite des Lieux de savoir, enfin, qui prendra une forme différente, pourra proposer une déclinaison de supports pour différents publics, c'est-à-dire pour un public international de chercheurs, pour un public scolaire, mais aussi pour le grand public, qui accédera, au moyen d'applications, à un univers savant sous une forme interactive et ludique. On peut parfaitement imaginer, pour ne citer qu'une possibilité, une mappemonde, sur laquelle on pourrait associer la Californie, l'Afrique et le Japon, pour voir ce que cette association permet de traverser dans l'histoire des savoirs et des traditions savantes. Voilà quelques exemples de modes de communication vers le grand public. Je suis aussi très favorable à l'open access et à des initiatives comme celles de revues.org ou hypotheses.org, en vue d'un partage et d'une communication aussi larges que possible, et en particulier non seulement pour le public européen, mais aussi pour les pays en développement. Je signale également une collection que nous avons lancée sur OpenEdition, “L’encyclopédie numérique ${ }^{25}$ », dont plusieurs volumes ont déjà paru. L’idée de cette “encyclopédie numérique» est aussi de partager le plus largement possible les contenus scientifiques.

25 La collection (http://books.openedition.org/oep/r27?lang=fr) est dirigée par M.Dacos et C.Jacob. Quatre volumes ont déjà paru: O. Ertzscheid, Qu'est-ce que l'identité numérique?, 20I3; С. ЈАсов, Qu'est-ce qu'un lieu de savoir?, 20I4; L. Burnard, What is the Text Encoding Initiative?, 20I4; J.-F. BERT, Qu'est-ce qu'une archive de chercheur?, 20I4. Plusieurs volumes sont en préparation. 